\title{
ERRATUM
}

\author{
V. Jacob · P. Jacob - R. Meckbach - S. A. Romanov
}

E. K. Vasilenko

\section{Lung cancer in Mayak workers: interaction of smoking and plutonium exposure}

Published online: 5 February 2006

(C) Springer-Verlag 2006

\section{Radiat Environ Biophys (2005) 44(2):119-129}

Unfortunately, the article was published with some errors. The correct parts are given here.

1. First sentence of paragraph before equation (2): Let $s$ be the smoking status ( 1 if smoking, 0 if not smoking), $d_{\mathrm{ext}, j}$ and $d_{\mathrm{int}, j}$ the annual external and internal dose in the $j$-th year of exposure, respectively, and $b y$ the year of birth.

2. Equation (9):

$\delta_{i}=\left\{\int_{t_{m i}}^{t_{2 i}}\left[\left(h_{i}(t) / h_{b, i}(t)-1\right) / D_{i}\left(t-t_{\mathrm{lag}}\right)\right] \mathrm{d} t\right\} /\left(t_{2 i}-t_{m i}\right)$,

3. Last paragraph of section Model parameters in the Chapter Discussion: the upper confidence bound -11.2 of $q_{0}^{\prime}$ implies an upper bound of the malignant transformation rate $\mu_{0}$ of $1.4 \times 10^{-5} \mathrm{a}^{-1}$. This value is consistent with the range of upper bounds $\left(3 \times 10^{-6}\right.$ $1 \times 10^{-4} \mathrm{a}^{-1}$ ) as derived from other analyses of lung cancer with the TSCE model [16].

4. Table 5:

The online version of the original article can be fount at http:// dx.doi.org/10.1007/s00411-005-0012-5

V. Jacob · P. Jacob $(\bowtie) \cdot$ R. Meckbach

Institute of Radiation Protection, GSF-National Research Center for Environment and Health, 85764 Neuherberg, Germany

E-mail: jacob@gsf.de

Tel.: + 49-89-31874008

Fax: + 49-89-31873363

Present address: V. Jacob

Klinikum rechts der lsar, Munich, Germany

S. A. Romanov

Southern Urals Biophysics Institute, Ozyorsk,

456780, Russian Federation

E. K. Vasilenko

Mayak Production Association, Ozyorsk,

456780, Russian Federation
Table 5 Fit parameters for models 1 and 2: best estimates, $68 \%$ confidence ranges, and type of distribution assumed in the calculation of the excess relative risk

\begin{tabular}{|c|c|c|}
\hline Fit parameter & Model 1 & Model 2 \\
\hline$X_{0}^{\prime}{ }^{\mathrm{a}}$ & $-16.0(-16.9 ;-15.2) \mathrm{N}$ & $-17.9(-18.8 ;-17.0) \mathrm{N}$ \\
\hline$X_{\mathrm{sm}}$ & - & $8.8(6.1 ; 13.1) \mathrm{LN}$ \\
\hline$X_{\text {ext }}\left(\right.$ year $\left.\mathrm{Sv}^{-1}\right)$ & $6.0(-0.1 ; 15.1) \mathrm{N}$ & $1.0(-13.9 ; 18.7) \mathrm{N}$ \\
\hline$X_{\text {int }}\left(\text { year } \mathrm{Sv}^{-1}\right)^{\mathrm{b}}$ & - & $54.6(33.5 ; 80.1) \mathrm{N}$ \\
\hline$X_{\text {by }}$ & $0.096(0.044 ; 0.232) \mathrm{LN}$ & $0.056(0.021 ; 0.142) \mathrm{LN}$ \\
\hline$\gamma_{0}\left(\right.$ year $\left.^{-1}\right)$ & $0.0804(0.0647 ; 0.0955) \mathrm{N}$ & $0.142(0.129 ; 0.156) \mathrm{LN}$ \\
\hline$\gamma_{\mathrm{sm}}\left(\right.$ year $\left.^{-1}\right)$ & $0.0616(0.0526 ; 0.0718) \mathrm{LN}$ & - \\
\hline$\gamma_{\text {lev }}\left(\right.$ year $\left.^{-1}\right)$ & $0.422(0.344 ; 0.528) \mathrm{LN}$ & - \\
\hline$\gamma_{\text {int }}\left(\mathrm{Sv}^{-1}\right)^{\mathrm{b}}$ & $0.107(0.089 ; 0.129) \mathrm{LN}$ & - \\
\hline & $-11.7(-12.2 ;-11.2) \mathrm{N}$ & $-11.5(-12.1 ;-10.8) \mathrm{N}$ \\
\hline$\mu_{\text {int }}\left(\text { year } \mathrm{Sv}^{-1}\right)^{\mathrm{b}}$ & - & $1.17(0.54 ; 2.47) \mathrm{LN}$ \\
\hline
\end{tabular}

$N$ normal, $L N$ lognormal distribution

${ }^{a} \operatorname{Exp}\left(X_{0}^{\prime}\right)$ has unit year ${ }^{-2}$

${ }^{\mathrm{b}}$ Assuming a radiation weighting factor of 20 for plutonium

${ }^{\mathrm{c}} \operatorname{Exp}\left(q_{0}^{\prime}\right)$ has unit year ${ }^{-1}$ 